\title{
ULTRASONS ET PHONONS DANS LES SUPRACONDUCTEURS
}

\author{
P. G. DE GENNES \\ Faculté des Sciences, Laboratoire de Physique des Solides, \\ associé au CNRS. 91 - Orsay, France
}

\begin{abstract}
Résumé. - On passe en revue, à un niveau élémentaire, les propriétés les plus marquantes des vibrations élastiques dans les métaux supraconducteurs :

a) faiblesse des changements de coefficients élastiques à la transition $\left(T=T_{\mathrm{c}}\right)$;

b) forte réduction de l'atténuation pour $T<T_{\mathrm{c}}$ et rôle des facteurs de cohérence ;

c) effets possibles en présence de lignes de tourbillon.

Enfin, on discute le principe des expériences d'effet tunnel qui permettent de déterminer le spectre des fréquences de phonons dans les supraconducteurs.

Abstract. - Some properties of elastic vibrations in superconductors are reviewed at an introductory level, including :

a) the weaknesses of the changes in elastic moduli at the transition temperature $T_{\mathrm{c}}$;

$b$ ) the strong decrease in ultrasonic attenuation when $T$ goes below $T_{c}$ and the importance of coherence factors ;

c) the various effects which may occur in the presence of vortices.

Finally, the principle of tunneling experiments which measure the phonon frequency spectrum in superconductors is presented.
\end{abstract}

I. Introduction. - Cet exposé est destiné à servir d'introduction pour des physiciens intéressés aux propriétés élastiques des solides, mais sans formation préalable en supraconductivité. Partons de quelques concepts de base [1].

a) L'ÉTAT FONDAMENTAL d'un métal supraconducteur est obtenu en associant les électrons par paires: pour un métal pur. par exemple, si nous avons un électron d'impulsion $\hbar k$, de spin $\uparrow$, nous en avons certainement un en - $k \downarrow$; si nous n'avons pas d'électron en $k$, nous n'en avons certainement pas en $-k \downarrow$.

Appelons $v_{k}^{2}$ la probabilité de trouver la paire $k \uparrow-k \downarrow$.

Appelons $u_{k}^{2}=1-v_{k}^{2}$ la probabilité de ne pas trouver la paire $k \uparrow-k \downarrow$.

L'aspect de $v_{k}^{2}$ est représenté sur la figure 1.

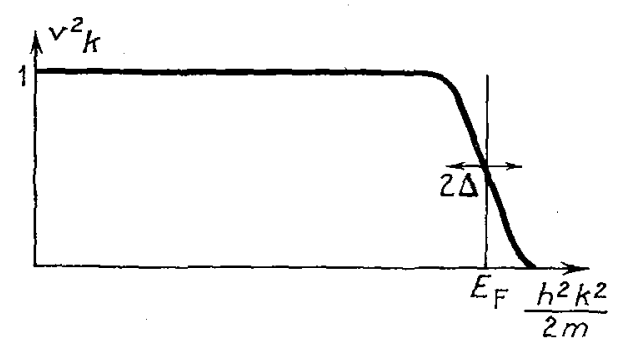

Fig. 1
Dans un métal normal, à $T=0, v_{k}^{2}$ passe abruptement à 0 quand on traverse la surface de Fermi, mais il y a un étalement sur un intervalle $A \sim k_{B} T_{c}$ en énergie. Il faut noter que $\Delta \ll E_{\mathrm{F}}$ dans tous les cas pratiques.

b) Les excitations individuelles sont obtenues en détruisant des paires, et cette opération implique un seuil d'énergie 4 . Plus précisément une excitation de vecteur d'onde $k$ a une énergie :

$$
\begin{aligned}
& \varepsilon_{k}=\sqrt{\Delta^{2}+\xi_{k}^{2}} \\
& \xi_{k}=\frac{h^{2}}{2 m} k^{2}-E_{\mathbf{F}}
\end{aligned}
$$

énergie d'excitation dans la phase normale.

$\mathrm{La}$ densité d'états des excitations

$$
\partial N / \partial \varepsilon=\partial N / \partial \xi \partial \xi / \partial \varepsilon
$$

diffère de la densité d'états dans la phase normale $\partial N / \partial \xi$ par un facteur

$$
\frac{\partial \xi}{\partial \varepsilon}=\frac{\Delta}{\sqrt{\varepsilon^{2}-\Delta^{2}}}
$$

Elle est représentée sur la figure 2. En général $A \sim 1,7 k_{\mathrm{B}} T_{\mathrm{c}}$ où $T_{\mathrm{c}}$ est la température de transition, $\Delta$ est mesuré par de nombreuses techniques [2], notamment les expériences d'effet tunnel dissipatifs 


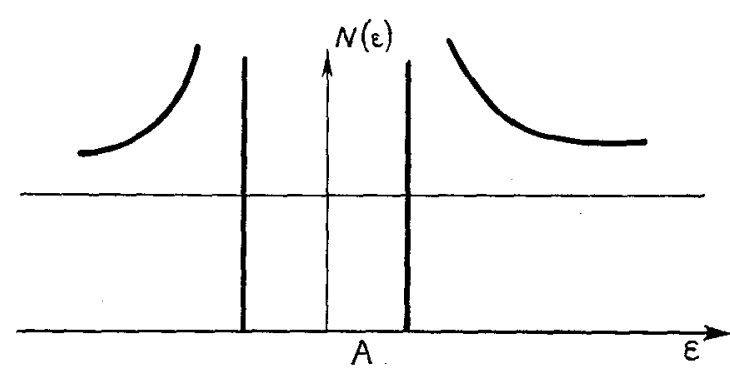

FIG. 2

inventées par I. Giaever en 1960, et représentées sur la figure 3 : un métal normal $N$ et un métal supraconduc-

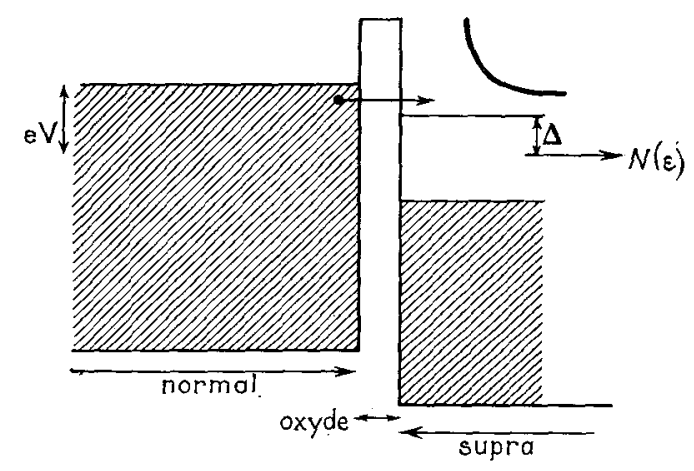

FIG. 3

teur $S$ sont séparés par une jonction isolante (épaisseur $\sim 30 \AA$ ) de résistance typique $1 \mathrm{ohm} . \mathrm{cm}^{2}$, comme le montre la figure, le courant est de la forme

$$
\begin{aligned}
I & =\operatorname{Cte} \times \int_{0}^{e V} N(\varepsilon) \mathrm{d} \varepsilon \quad\left(k_{\mathrm{B}} T \ll \Delta\right) \\
\frac{\mathrm{d} I}{\mathrm{~d} V} & =\operatorname{Cte} N(\mathrm{eV})
\end{aligned}
$$

d'où une caractéristique $I(V)$ qui a l'aspect montré sur la figure 4 , sur laquelle on lit immédiatement la valeur de $\Delta$.

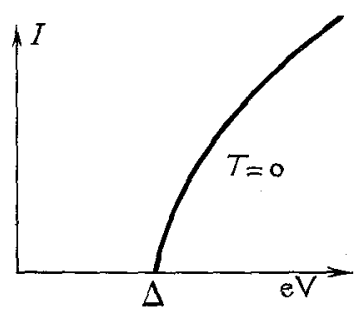

FIG. 4
La variation en température de $\Delta$, déduite de la théorie, a l'aspect représenté sur la figure 5.

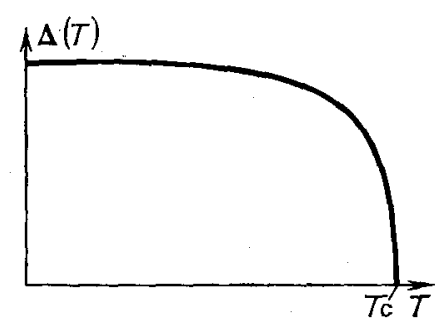

FiG. 5

II. Effets de la transition supraconductrice sur les propriétés élastiques. - $a$ ) EFFETS SUR LA RELATION DE DISPERSION $\omega(q)$ DES PHONONS. Ils sont très faibles. Par exemple les constantes élastiques montrent au point de transition $T_{\mathrm{c}}$ une discontinuité dont l'amplitude relative est $\sim 10^{-6}$ [3]. La vitesse du son est aussi pratiquement inchangée.

Ce résultat peut surprendre : la condensation en paires que nous avons décrite pour les électrons se retrouve dans un autre cas physique : celui des neutrons et protons d'un noyau lourd. Dans ce dernier cas, les propriétés ( mécaniques ) du noyau (déformation ellipsoïdale, moments d'inertie) sont extrêmement sensibles à l'appariement [4].

Pour le gaz d'électrons, l'absence d'effets peut être comprise par le raisonnement suivant : l'énergie de liaison est une somme de contributions faisant intervenir tous les électrons à l'intérieur de la mer de Fermi. La probabilité $v_{k}^{2}$ de trouver un électron dans un état $k$ est arrondie sur un intervalle $\Delta$ en énergie comme le montre la figure 1 , mais on garde le même nombre total d'électrons $\sum v_{k}^{2}=$ Cte. Dans ces conditions, l'énergie de liaison $E_{\varphi}$ n'est modifiée que par des termes $\sim\left(\Delta / E_{\mathrm{F}}\right)^{2} \lesssim 10^{-6}$. La même dépendance se retrouve pour les compressibilités (dérivées de $E_{\varphi}$ par rapport au volume). Le seul cas où un effet notable peut survenir est celui des composés du type $\mathrm{Nb}_{3} \mathrm{Sn}$, pour lesquels il y a une bande $d$ très étroite [5,] [6] $\left(E_{F} \sim 300 \circ \mathrm{K}\right)$, donc des constantes élastiques très sensibles à la température.

Pour les noyaux lourds, la situation est différente : les déformations et les moments d'inertie sont contrôlés par l'état des nucléons dans la dernière couche incomplète. L'effet de $\Delta(\sim 0,5 \mathrm{MeV})$ dans cette couche est fort.

On peut se demander s'il y a des effets plus spectaculaires pour des vibrations $\omega_{q}$ de courtes longueurs d'onde, et en particulier dans la région de l'anomalie de Kohn [7] $q=2 k_{\mathrm{F}}$. Le caractère arrondi de la 
distribution $v_{k}^{2}$ tend à diminuer cette anomalie. Mais comme $\Delta \sim k_{\mathrm{B}} T_{\mathrm{c}}$ cet effet $n$ 'est pas plus grand que l'arrondissement dû à la largeur finie de la distribution de Fermi, dans la phase normale, pour $T \sim T_{\mathbf{c}}$. En pratique, ici encore, on ne peut espérer un effet spectaculaire de la température sur l'anomalie de Kohn que (peut-être) dans $\mathrm{Nb}_{3} \mathrm{Sn}$ et les composés analogues.

b) Atténuation ultrasonore : FaCteurs de COHÉRENCE. - La partie imaginaire des constantes élastiques, associée aux effets d'atténuation montre des effets beaucoup plus spectaculaires. Dès 1955 Bommel [8] a observé une chute brutale de l'atténuation pour les ondes longitudinales dans $\mathrm{Pb}$ et $\mathrm{Sn}$ monocristallin (Fig. 6), lorsque $T$ devient inférieur à $T_{\mathrm{c}}$. Au
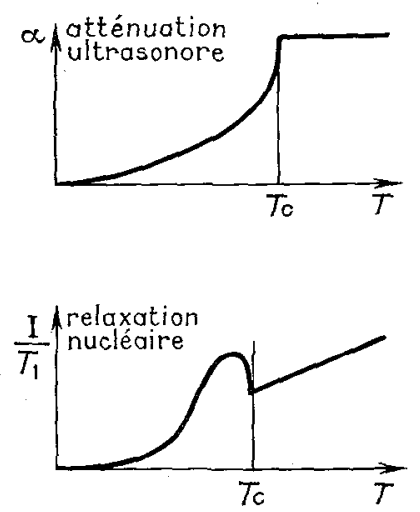

FIG. 6.

premier abord, cet effet paraît presque trivial : le nombre d'excitations présentes tombe rapidement (comme $\mathrm{e}^{-\Delta / k} \mathrm{~B}^{T}$ lorsque $k_{B} T<\Delta$ ) et tous les mécanismes dissipatifs varient en conséquence. Mais on voit la nécessité d'une explication un peu plus subtile si l'on compare des effets dissipatifs différents tels que atténuation ultrasonore et relaxation nucléaire (Fig. 6 et 7). Pour ce dernier phénomène, on observe que le taux de relaxation commence par croître quand $T$ décroît et traverse $T_{\mathrm{c}}$.

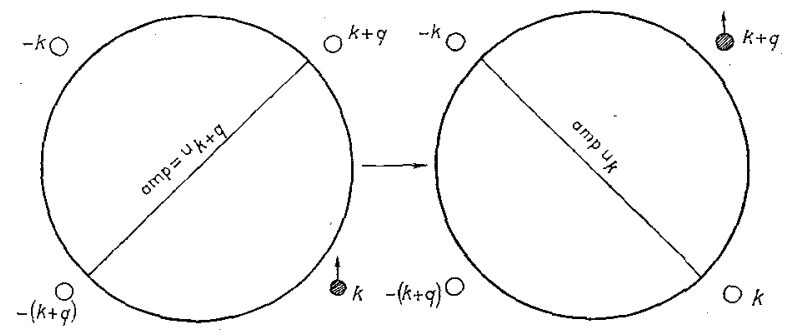

FIG. $7 a$.

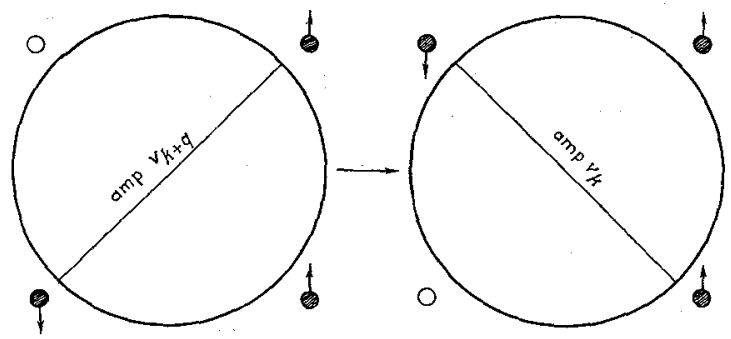

FIG. $7 b$.

L'explication de cette différence est un des succès majeurs de la théorie de Bardeen, Cooper et Schrieffer (BCS) [1]. Son principe est donné sur la figure 7. Considérons une collision d'un électron sur une vibration de réseau ( $\rightarrow$ atténuation ultrasonore) ou sur un spin nucléaire $(\rightarrow$ relaxation nucléaire). Soit $\hbar q$ l'impulsion gagnée par les électrons dans cette collision. Une façon possible de la réaliser est montrée sur la figure $7 a:$ un électron non apparié passe de $k \uparrow$ à $k+q \uparrow$. L'amplitude de probabilité correspondante $A_{1}$ contient un facteur $u_{k+q}$ spécifiant que les cases $(k+q),-(k+q)$ sont initialement vides, et un facteur $u_{k}$ spécifiant que les cas $k_{1}-k$ sont vides dans l'état final.

$$
A_{1}=u_{k+q} u_{k}(k+q \uparrow|V| k \uparrow)
$$

où $(k+q \uparrow|V| k \uparrow)$ est un élément de matrice de la perturbation entre états à 1 électron (interaction électron-phonon ou interaction électron-spin nucléaire).

Mais ce processus interfère avec celui de la figure $7 b$ où l'électron $-(k+q) \downarrow$ initialement engagé dans une paire avec l'électron célibataire $k \uparrow$. Ici l'amplitude est

$$
A_{2}=-v_{k+q} v_{k}(-k \downarrow|V|-k-q \downarrow) .
$$

Le signe - vient de l'antisymétrie des fonctions d'ondes : la composante de la fonction d'onde que nous utilisons dans l'état initial est un déterminant de Slater à 3 particules

$$
\left.v_{k+q} \mid-(k+q) \downarrow, k+q \uparrow, k \uparrow\right)
$$

et dans l'état final

$$
\left.v_{k} \mid-k \downarrow, k \uparrow, k+q \uparrow\right)
$$

mais la perturbation $V$ agissant sur la première donne

$$
\text { Cte } x \mid-k \downarrow,(k+q) \uparrow, k \uparrow)
$$

qui diffère par une permutation de deux colonnes, donc par un signe - , de la précédente.

Pour obtenir l'amplitude totale on doit superposer $A_{1}$ et $A_{2}$. 
Dans les cas qui nous intéressent les collisions sont presque élastiques (par exemple pour l'atténuation ultrasonore, on travaille en général avec des phonons de fréquence $\left.\omega_{q} \ll \Delta / \hbar\right)$. Comme $u_{k}$ ne dépend que de l'énergie $\varepsilon_{k}$, si $\varepsilon_{k}=\varepsilon_{k+q}, u_{k+q}=u_{k}, v_{k+q}=v_{k}$ l'amplitude est

$$
\begin{aligned}
A=A_{1}+A_{2}=u_{k}^{2}(k+ & q \uparrow|V| k \uparrow)- \\
& -v_{k}^{2}(-k \downarrow|V|-k-q \downarrow) .
\end{aligned}
$$

$1^{\circ}$ Cas ultrasonore : l'interaction électron-phonon est indépendante du spin et les deux éléments de matrice sont égaux à une même constante $V_{q}$. La probabilité de transition, et finalement l'atténuation $\alpha$, sont de la forme

$$
\alpha=\operatorname{Cte} \int_{\Delta}^{\infty} N^{2}(\varepsilon) \mathrm{d} \varepsilon|A|^{2} f(\varepsilon)(1-f(\varepsilon))
$$

$N(\varepsilon)$ est la densité d'états des excitations, $f(\varepsilon)$ la fonction de Fermi

$$
f(\varepsilon)=\frac{1}{\mathrm{e}^{\varepsilon / k_{\mathrm{B}} T}+1} .
$$

Les facteurs $f(\varepsilon)$ et $1-f(\varepsilon)$ sont les facteurs habituels pour les collisions d'excitations obéissant à la statistique de Fermi.

$N(\varepsilon)$ a un pic pour $\varepsilon=\Delta$ mais $A \sim\left|u^{2}-v^{2}\right|$ s'annule pour $\xi_{k}=0 . \varepsilon=2 \Delta$ comme le montre la figure 1 . Au total le produit $|N(\varepsilon) A|^{2}$ se réduit juste à une constante et

$$
\alpha=\operatorname{Cte} \int_{\Delta}^{\infty} f(\varepsilon)(1-f(\varepsilon)) .
$$

Comme

$$
\frac{\partial f}{\partial \varepsilon}=-\frac{1}{k_{\mathrm{B}} T} f(1-f)
$$

on peut intégrer cette formule. Si $V_{q}$ est indépendant de la température et si $\alpha_{n}$ désigne l'atténuation dans la phase normale, on trouve ainsi

$$
\frac{\alpha}{\alpha_{n}}=2 f(\Delta) \text {. }
$$

Cette formule, cumulée avec la forme théorique de variation de $\Delta$ avec $T$ (Fig. 5) décrit assez bien l'atténuation mesurée pour les ondes longitudinales (Fig. 6).

$2^{\circ}$ Cas de la relaxation nucléaire. Ici l'interaction $V$ a la forme $A I S$ où $A$ mesure le couplage hyperfin et les éléments de matrice entre états électroniques de spin $\uparrow$ (ou $\downarrow$ ) sont respectivement $\pm A I_{z} \frac{1}{2}$.
Il y a interfẻrence constructive entre $A_{1}$ et $A_{2}$, le facteur $N^{2}(\varepsilon)$ qui est grand pour $\varepsilon$ petit n'est plus compensé, et la relaxation nucléaire prend la forme :

$$
\frac{1}{T_{1}}=\text { const. } \int_{\Delta}^{\infty} N^{2}(\varepsilon) f(\varepsilon)(1-f(\varepsilon)) \mathrm{d} \varepsilon
$$

Quand $T$ est peu au-dessous de $T_{\mathrm{c}}$, le pic qui apparaît dans $N(\varepsilon)$ augmente la vitesse de relaxation. Pour $T \ll T_{\mathrm{c}}$ le facteur $f(\varepsilon)$ l'emporte et $1 / T_{1}$ devient petit, d'où l'aspect de la figure 7 .

Au total la différence observée entre ces deux effets dissipatifs montre clairement que la strueture $k \uparrow-k \downarrow$ proposée par BCS pour les paires est correcte. Les facteurs cruciaux $\left(u_{k} u_{k+q} \mp v_{k} v_{k+q}\right)$ portent le nom de facteurs de cohérence [1].

c) Cas des ondes transversales. - Dans un métal assez propre (libre parcours moyen électronique $l>$ longueur d'onde acoustique $2 \pi / q):$ l'atténuation des ondes transversales n'obéit pas à la loi $\alpha / \alpha_{n}=2 f(\Delta)$ mais décroît très brutalement quand $T$ décroît et traverse $T_{\mathrm{c}}$ (Fig. 8). L'atténuation subit une dis-

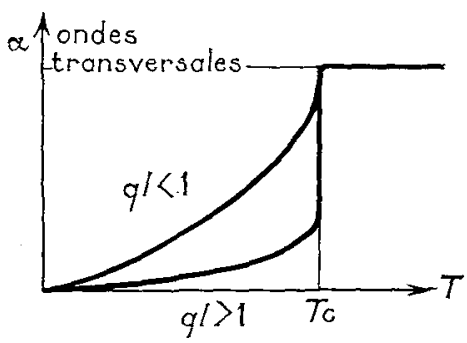

FIG. 8.

continuité à $T_{\mathrm{c}}$. Par contre, pour $q l<1$ on a la même forme que pour une onde longitudinale. $\mathrm{Ce}$ comportement a été étudié notamment par Claiborne sur $\mathrm{Al}$ et il a aussi proposé une théorie simple qui en rend compte [9].

$1^{\circ}$ Dans la phase normale le courant électronique $j_{e}$ est lié au champ électrique $E$ et à la vitesse de déplacement élastique $u$ par

$$
j_{e}=\sigma(\mathrm{q})\left(E-\frac{m u}{e \tau}\right)
$$

$\sigma(q)$ est la conductivité pour une perturbation électrique de longueur d'onde $2 \pi / q \cdot \tau=l / v_{\mathrm{F}}$ est le temps de collision électrons impuretés. Le terme $-m u / \tau$ représente la force d'entraînement due aux impuretés qui se déplacent à la vitesse $u$ ( $m=$ masse électronique).

En pratique, aux fréquences qui nous concernent, 
le courant $j_{e}$ équilibre exactement le courant dû aux ions positifs.

$$
j_{e}=-n e u
$$

( $n=$ nombre d'électrons $\left./ \mathrm{cm}^{3}\right)$.

Donc

$$
E=n e u\left[\frac{1}{\sigma(0)}-\frac{1}{\sigma(q)}\right]
$$

ou $\sigma(0)=n e^{2} \tau / m$ est la conductivité habituelle. La puissance dissipée par $\mathrm{cm}^{+}$est $1 / 2 \operatorname{Re}\left\{E \cdot j_{\mathrm{e}}^{+}\right\}$et le coefficient d'atténuation

$$
\alpha=\frac{\operatorname{Re}\left\{\boldsymbol{E} \cdot j_{\mathrm{e}}^{+}\right\}}{\rho|u|^{2}}=\frac{m n}{\rho s \tau}\left(-1+\frac{\sigma(0)}{\sigma(q)}\right)
$$

( $\rho=$ densité du solide, $s=$ vitesse du son).

Pour une discussion détaillée de cette formule et ses conséquences, voir Pippard [10].

$2^{\circ}$ Dans la phase supraconductrice le courant $j_{\mathrm{e}}$ comprend un courant superfluide $j_{\mathrm{s}}$ et un courant ( normal ) (dû aux excitations) $j_{\mathrm{n}}$.

$$
j_{\mathrm{n}}=\sigma_{\mathrm{n}}(q)\left(E-\frac{m u}{e \tau}\right)
$$

où $\sigma_{\mathrm{n}}(q)$ est proportionnel au nombre d'excitations présentes (Claiborne prend $\sigma_{\mathrm{n}}(q)=\sigma(q) 2 f(\Delta)$ ).

Le champ électrique transversal $E$ est dû à un effet d'induction

$$
\operatorname{rot} E=-\frac{1}{c} \frac{\partial H}{\sigma t}
$$

Or, dans un supraconducteur, un champ magnétique faible est toujours expulsé (effet Meissner) $\boldsymbol{H}=\mathbf{0}$ donc $E_{\text {transversal }}=0$

$$
j_{\mathrm{n}}=\sigma_{\mathrm{n}}(q)\left(-\frac{m u}{e \tau}\right) .
$$

Introduisons la vitesse $v_{\mathrm{n}}$ et la concentration $n_{\mathrm{n}}$ du (c fluide normal » par les formules

$$
\begin{aligned}
j_{\mathrm{n}} & =-n_{\mathrm{n}} v e_{\mathrm{n}} \\
\sigma_{\mathrm{n}}(0) & =n_{\mathrm{n}} \frac{e^{2} \tau}{m} .
\end{aligned}
$$

Alors

$$
v_{\mathrm{n}}=u \frac{\sigma_{\mathrm{n}}(q)}{\sigma_{\mathrm{n}}(0)}<u
$$

Ici la dissipation ne provient plus de $\boldsymbol{E} . j_{e}$ mais uniquement de la présence d'impuretés : à chaque colli- sion le fluide normal cède au réseau une impulsion moyenne $m\left(-u+v_{\mathrm{n}}\right)$ donc la puissance dissipée est

$$
\begin{array}{r}
1 / 2 \operatorname{Re}\left(u^{*} n_{\mathrm{n}} m / \tau\left(-v_{\mathrm{n}}+u\right)\right) \\
\alpha=\frac{m^{2}}{\rho s \tau^{2} e^{2}}\left(\sigma_{\mathrm{n}}(0)-\sigma_{\mathrm{n}}(q)\right)
\end{array}
$$

soit en comparant au cas normal

$$
\frac{\alpha}{\alpha_{\mathrm{n}}}=\frac{\sigma_{\mathrm{n}}(q)}{n e^{2} \tau / m}=2 f(\Delta) \frac{\sigma(q)}{\sigma(0)} .
$$

On attend une atténuation $\alpha$ encore proportionnelle à $2 f(4)$. Mais il y a en général une discontinuité de $\alpha$ à $T_{\mathrm{c}}$

$$
\begin{aligned}
& \left(\frac{\alpha}{\alpha_{\mathrm{n}}}\right)_{T=T_{\mathrm{c}}+0}=1 \\
& \left(\frac{\alpha}{\alpha_{\mathrm{n}}}\right)_{T=T_{\mathrm{c}}-0}=\frac{\sigma(\mathrm{q})}{\sigma(0)}<1 \text { si } q l \text { n'est pas trop petit. }
\end{aligned}
$$

Les résultats de Claiborne sur $A_{1}$ sont en bon accord avec cette théorie simple. Il a aussi montré que, en réalité, la discontinuité est étalée sur un intervalle $T_{\mathrm{c}}-T \sim 1 / 100^{\circ} \mathrm{K}$ (dans cet intervalle il y a encore trop peu d'électrons supraconducteurs pour réaliser un effet Meissner complet). La théorie a été formulée dans un langage plus microscopique par Tsuneto [11] avec essentiellement les mêmes résultats.

d) INFORMATIONS DÉDUITES DES MESURES D'ATTÉNUATION. - $1^{\circ}$ Pour des ondes longitudinales on peut, à partir des valeurs mesurées de

$$
\frac{\alpha}{\alpha_{n}}=\frac{2}{\mathrm{e}^{\Delta / k_{\mathrm{B}} T}+1}
$$

déterminer la valeur et la variation en température de $\Delta$. Ces études, faites en particulier par Morse et ses collaborateurs [9], donnent bien en $1^{\text {re }}$ approximation la forme de $\Delta(T)$ attendue d'après la théorie BCS. Elles font apparaître aussi des effets plus fins : le paramètre $\Delta$ défini par l'énergie d'excitation

$$
\varepsilon_{k}=\sqrt{\xi_{k}^{2}+|\Delta|^{2}}
$$

varie en fait sur la surface de Fermi. Ces variations, dont l'amplitude peut atteindre $30 \%$, sont refletées par une anisotropie du rapport $\alpha / \alpha_{\mathrm{n}}$ en fonction de la direction de propagation $q$ (dans un monocristal). Comme pour un métal normal, les excitations qui sont bien couplées à l'onde acoustique sont celles dont la vitesse $v$ (dans la direction $q$ ) est égale à la vitesse du son $s$, donc très faible par rapport à la vitesse de Fermi 
$v_{F}$. Pour une surface de Fermi sphérique par exemple, elles sont localisées au voisinage du plan équatorial perpendiculaire à $q$ (Fig. 9). La valeur de $\Delta$ qui est mesu-

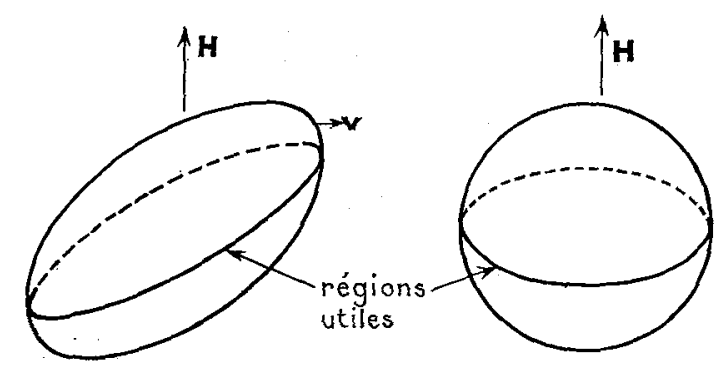

FIG. 9.

rée représente une moyenne sur le cercle équatorial. Actuellement ces mesures ultrasonores nous donnent les meilleurs renseignements disponibles sur l'anisotropie de $\Delta$. Il se peut toutefois que dans l'avenir des mesures d'effet tunnel sur monocristaux permettent de déterminer $\Delta$ pour une direction $k$ (et non plus une moyenne sur une courbe équatoriale).

$2^{\circ}$ Dans certains métaux supraconducteurs $\alpha$ est assez sensible au niveau de puissance ultrasonore. Pour le plomb, les effets non linéaires apparaissent seulement dans la phase supraconductrice. Ils ont été observés en premier par Love et Shaw [12], puis par Tittmann et Bommel [13] (Fig. 10). Ces derniers

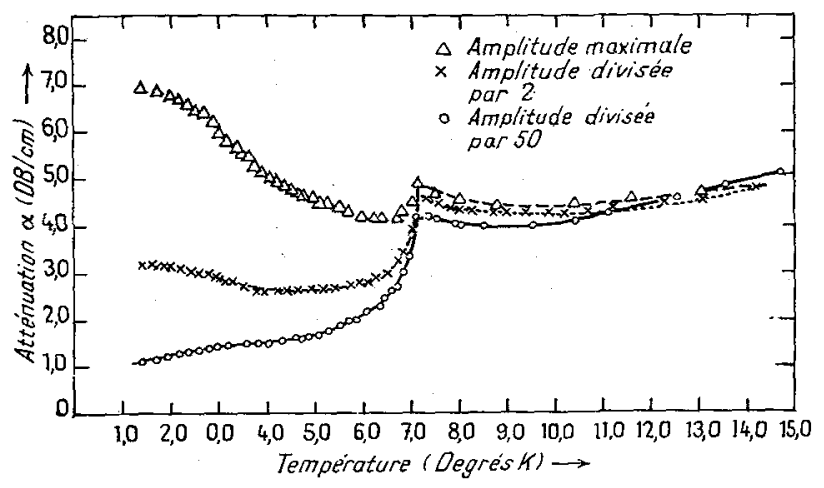

FrG. 10. - Influence de la température sur l'atténuation dans un cristal unique de plomb de pureté $99,999 \%$. Fréquence $51 \mathrm{Mc}$, ondes longitudinales de direction $\langle 111\rangle$ (d'après Tittmann et Bommel [13]).

ont proposé une interprétation ingénieuse: l'absorption non linéaire serait due à l'arrachage de dislocations ancrées sur des défauts faibles (impuretés ?). Dans la phase normale ces dislocations ne se déplaceraient pratiquement pas en présence de l'onde ultrasonore, car les électrons normaux leur imposent un fort amortissement visqueux. Mais dans la phase supraconductrice cet amortissement disparaît, les dislocations ont des mouvements de grande amplitude et l'atténuation non linéaire peut apparaître. Tittmann et Bommel ont montré qu'en ajoutant des impuretés on diminue les effets non linéaires (ancrage meilleur) et qu'en augmentant le nombre de dislocations on augmente d'abord l'effet non linéaire, puis on passe par un maximum (les dislocations s'ancrant fortement les unes sur les autres). Il reste à expliquer comment les dislocations parviennent à se détacher des impuretés aux très basses températures qui sont ici en cause.

$3^{\circ}$ Un domaine particulièrement intéressant pour l'avenir est celui de l'atténuation ultrasonore dans les matériaux dits ( đe $2^{\mathrm{e}}$ espèce ) sous champ magnétique. (Dans ces matériaux, le champ pénètre dans la masse, sous forme de (clignes de tourbillon ) [14].) En pratique, il y a une difficulté : la plupart des matériaux de $2^{\mathrm{e}}$ espèce sont des alliages, où la contribution des électrons de conduction à l'atténuation est faible et souvent masquée par des effets parasites. Mais des mesures exploratoires ont pu être faites sur un monocristal de niobium [15]. Les principaux effets attendus sont les suivants :

a) Le spectre des excitations dans l'état tourbillonnaire est profondément modifié comme le montrent les expériences d'effet tunnel [16] et les calculs théoriques [17]. Ceci a évidemment un fort retentissement sur l'atténuation : cette question sera discutée ici par Cyrot.

b) Il y a peut-être des résonances géométriques lorsque la longueur d'onde ultrasonore est égale à la distance entre tourbillons [18]. Ceci exige toutefois des mesures à très hautes fréquences $(10000 \mathrm{Mc})$.

c) Dans un métal de $2^{\mathrm{e}}$ espèce très pur, les lignes de tourbillon ont probablement des vibrations collectives [19]. Si l'existence de ces vibrations se confirme, il pourra dans certains cas [19] y avoir un fort couplage entre elles et une vibration acoustique. En outre, on peut imaginer, avec les tourbillons, des effets non linéaires analogues à ceux observés par Tittmann et Bommel et attribués aux dislocations.

III. Informations sur les spectres de phonons obtenues par effet tunnel. - Nous avons vu dans la section I comment la mesure d'une caractéristique tunnel, à basse température, permet de déterminer la densité d'états $N(\varepsilon)$ des excitations. Dans les expériences usuelles, les voltages auxquels on s'intéresse sont tels que $e V \sim \Delta$ (typiquement 1 millivolt). Si on a la curiosité de pousser les mesures vers des voltages 
un peu plus élevés ( $\sim 10$ millivolts) on voit souvent apparaître des anomalies faibles mais intéressantes (voir figure 11).

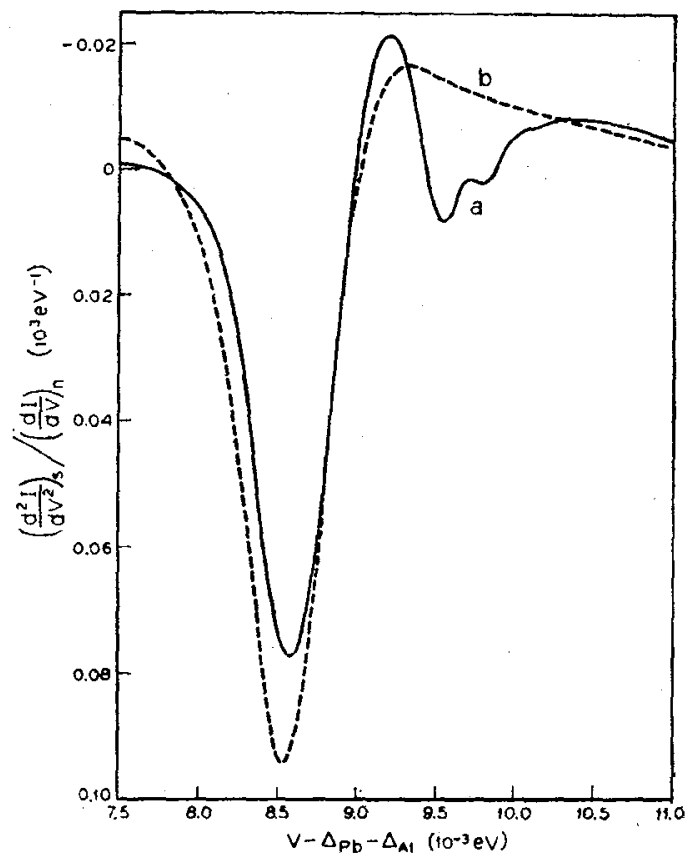

FIG. 11. - Courbe $a$ ) : dérivée seconde $d^{2} I / d V^{2}$ du courant de tunnel en fonction de la tension, pour une fonction tunnel $\mathrm{AlIPb}_{0,97} \mathrm{In}_{0,03}$ à $0,83{ }^{\circ} \mathrm{K}$ dans la région des énergies de phonon $\approx 9 \times 10^{-23} \mathrm{eV}$;

courbe $b$ ) : mêmes données pour une fonction $\mathrm{Al} / \mathrm{Pb}$ (d'après Rowell [23]).

Des calculs détaillés ont été faits pas Schrieffer et al. [20] pour rendre compte de ces effets. Le principe de l'explication est simple [21]. Une excitation d'énergie $\varepsilon$ peut émettre un phonon de fréquence $\Omega$; et aboutir à l'énergie finale $\varepsilon^{\prime}$. Ces processus donnent une correction de l'énergie de notre excitation, et aussi une durée de vie finie. Les deux effets réagissent sur la densité d'états. Considérons par exemple le second : pour une collision réelle nous avons $\varepsilon^{\prime}+\hbar \Omega=\varepsilon$. Le processus sera particulièrement important si $\Omega$ coïncide avec un pic $\Omega_{0}$, de la distribution des phonons $g(\Omega)\left(\Omega_{0}\right.$ singularité de Van Hove). Mais ceci ne suffit pas : dans un métal normal, par exemple, où les états finaux $\varepsilon$, ont une répartition très régulière, toute valeur de $\varepsilon$ conduit au même effet, et rien n'est observé sur $N(\varepsilon)$. Mais dans un supraconducteur $\varepsilon^{\prime}$ est très concentré au voisinage de $\Delta$ (voir la figure pour la densité d'états). On aura donc une anomalie dans $N(\varepsilon)$ pour

$$
\varepsilon=\hbar \Omega_{0}+\Delta .
$$

Il est donc possible de localiser les pics $\Omega_{0}$ de la distribution des phonons par une mesure d'effet tunnel. Ceci a été fait notamment [22] pour :

- l'étain ;

- le plomb : dans ce cas les spectres de phonons ont été directement mesurés par diffusion de neutrons, et l'accord entre les deux déterminations des pics $\Omega_{0}$ est bon.

Plus récemment, la méthode a permis de mettre en évidence l'existence de modes localisés en présence d'impuretés légères (In) dans $\mathrm{Pb}$ [23] (fig. 11).

Il est intéressant d'estimer l'épaisseur de mesure dans une telle expérience : la particule d'énergie $\varepsilon$ est injectée à partir de la jonction. A quelle distance de la jonction le phonon $\Omega_{0}$ est-il émis, ou absorbé ? Les calculs de Schrieffer et al. [20] montrent que le temps de vie $\tau$ de la particule est court si $\varepsilon / \hbar$ est comparable à la fréquence de Debye $\Omega_{\mathrm{D}}$. En fait, à un coefficient près, on a $\tau=\Omega_{\mathrm{D}}^{-1}$ pour de telles valeurs de $\varepsilon$. Le libre parcours moyen correspondant est $\sim v_{F} / \Omega_{\mathrm{D}}$ soit typiquement $300 \AA$. Des mesures d'effet tunnel sur doubles couches (jonction + métal d'épaisseur $\sim v_{F} / \Omega_{0}+2^{\mathrm{e}}$ métal) devraient donner des informations sur ces portées.

\section{Bibliographie}

[1] Pour une introduction claire et détaillée à la théorie de Bardeen, Cooper et Schrieffer, voir TinkHAM (M.), Cours de l'école d'été des Houches 1961 (Gordon and Breach, New York 1962), dans ( Low Temperature Physics》.

[2] Voir la revue par Douglass (D. H.) \& Falicov (L. M.) dans Progress in Low Temperature Physics, tome IV, édité par GoRTER (C. J.) (North Holland, Amsterdam 1964).

[3] Pour une analyse thermodynamique de la discontinuité de la compressibilité, voir LandaU (L.) et LIFSHTTZ (I. M.), Electrodynamics of continuous media, Chap. VI (Pergamon Press, New York 1960).

Pour une discussion des résultats expérimentaux, voir ANDRes (K.), Olsen (H.) et Rohrer (H.), IBM Journ., 1962, 6, 84.

[4] Voir par exemple Baranger (M.), Cours de l'école d'été de Cargèse 1962, édités par LevY (M.) (Benjamin, New York 1963).

[5] Weger (M.), Rev. Mod. Physics, 1964, 36, 175.

[6] LabBé (J.) et Friedel (J.), Instabilité électronique et changement de phase cristalline des composés du type $\mathrm{V}_{3} \mathrm{Si}$ à basse température, $J$. Physique, 1966, 27. 53.

[7] KoHn (W.), Phys. Rev. Lett., 1959, 2, 393.

[8] Bommel (H. E.), Phys. Rev., 96, 220.

[9] Voir MoRse (R. W.), IBM Journ., 1962, 6, 58.

[10] Pippard (A. B.), Cours de l'école des Houches 1961, «Low. Temperature Physics ». (Gordon et Breach, New York 1962). 
[11] Tsuneto (T.), Phys. Rev., 1961, 121, 402, et à paraître. [12] Love (R. E.), Shaw (R. W.), Rev. Mod. Physics, 1964, 36, 260.

[13] Tittmann (B. R.) et Bommel (H. E.), Phys. Rev. Lett. 1965, 14, 296.

[14] Abrikosov (A.), Soviet Physics J.E.T.P., 1957, 5, 1174.

[15] Ikushima (A.), FuJI (M.), Susuki (T.), J. Phys. Chem. Sol., 1965, 26.

[16] GuYon (E.), Thèse Orsay, 1965.

[17] Caroli (C.), De Gennes (P. G.) et Matricon (J.), Phys. Lett., 1964, 9, 307.

Caroli (C.) et Matricon (J.), Phys. Mat. Condensée, $1965,3,380$.

Сүrot (M.), Phys. Mat. Condensée, 1965, 3, 374.
De Gennes (P. G.), Phys. Mat. Condensée, 1965, 3, 79.

[18] Cooper (L.), Houghton (A.), Lee (H. J.), Phys. Rev. Lett., 1965, 15, 584.

[19] De Gennes (P. G.) et Matricon (J.), Rev. Mod. Phys., 1964, 36, 45.

[20] Schrieffer (R.), Scalapino (D. J.), Wilkins (J. W.), Phys. Rev. Lett., 1963, 10, 336.

[21] Scalapino (D. J.) et Anderson (P. W.), Phys. Rev., 1964, 133, A 921.

[22] Rowell (J. M.), ANDerson (P. W.), Thomas (D. E.), Phys. Rev. Lett., 1963, 10, 334.

Bermon (S.), Ginsberg (D. M.), Phys. Rev., 1964, 135, A 306.

[23] Rowell (J. M.), MC MILLAN (W. L.), ANDERson (P. W.), Phys. Rev. Lett., 1965, 14, 633. 